\title{
Severe Acute Respiratory Syndrome Coronavirus 2 (SARS-CoV-2) Pandemic: Is Sequela the Bigger Threat?
}

\author{
Nitin Rai ${ }^{1} \odot$, Dalim Kumar Baidya ${ }^{2}$ \\ Keywords: COVID-19, ICU mortality, Risk factor. \\ Indian Journal of Critical Care Medicine (2021): 10.5005/jp-journals-10071-23734
}

To the Editor,

The severe acute respiratory syndrome coronavirus-2 (SARS-CoV-2) pandemic has been affecting the population worldwide for over six months now. Despite having a recovery rate of about $80 \%$, around 32 to $55 \%$ of patients continue to have one or more symptoms at the 60-day follow-up period. ${ }^{1}$

While long-term follow-up studies of coronavirus disease (COVID-19) patients are still underway, post-COVID-19 pulmonary fibrosis is one such complication being faced by clinicians worldwide. The exact prevalence of post-COVID-19 fibrosis is not known, but it has been reported in more than one-third of recovered patients. ${ }^{2}$ Search from previous severe acute respiratory syndrome and Middle East respiratory syndrome epidemic has revealed length of intensive care unit stay, disease severity, hypertension, diabetes, smoking, and advanced age to be predictors of development of fibrosis.

Antifibrotics nintedanib and pirfenidone had been studied exclusively in idiopathic pulmonary fibrosis (IPF). The putative role in post-COVID fibrosis has been from the extrapolation of their role in chronic IPF. There are many caveats associated with the possible role of these drugs in mitigating fibrotic changes after COVID-19. The profibrotic pathway is a result of a variable mixture of immunologically mediated damage and classical acute lung injury. ${ }^{3}$ Antifibrotic therapy does not affect the immune dysregulation pathway, hence a combination with other anti-inflammatory drugs could be a rational approach.

Another problem is the formulation of antifibrotics. Both nintedanib and pirfenidone are available in oral preparation. The unpredictable absorption in critically ill patients and difficulty administering to patients on a ventilator along with delayed metabolism in patients having deranged liver function enzymes are some of the problems.

A further problem is the rapidity of action of these drugs. Patients treated for IPF are generally followed yearly to monitor the progress by evaluating the pulmonary function tests, especially forced vital capacity. ${ }^{4}$

In the current scenario, we do not have sufficient data on how well the patients have fared after treatment with these antifibrotics.

The optimal timing of initiating therapy also warrants some attention. There are no recommendations for prophylactic initiation of antifibrotic therapy in COVID-19 patients. The benefit
${ }^{1}$ Fortis Escorts Heart Institute, New Delhi, India
${ }^{2}$ All India Institute of Medical Sciences, New Delhi, India

Corresponding Author: Nitin Rai, Department of Critical Care Medicine, Fortis Escorts Heart Institute, New Delhi, India, Phone: +918527613852, e-mail: nitinrai311@gmail.com

How to cite this article: Rai N, Baidya DK. Severe Acute Respiratory Syndrome Coronavirus 2 (SARS-CoV-2) Pandemic: Is Sequela the Bigger Threat? Indian J Crit Care Med 2021;25(2):245-246.

Source of support: Nil

Conflict of interest: None

of antifibrotics is doubtful once the fibrotic changes are already established. Early experimental models have shown that starting antifibrotics in the early phase of lung injury promotes fibrosis and initiation in the later stages of injury at the onset of fibrotic phase might ameliorate fibrosis. ${ }^{5}$

Multiple profibrotic pathways lead to fibrosis. Apart from immunomodulatory pathway, there is evidence that vascular dysfunction involving vascular endothelial growth factor and tumor necrosis factor- $\alpha$ is a key component leading to fibrosis. The commonly used antifibrotics do not target every pathway leaving some profibrotic pathways to perpetuate despite ongoing therapy.

As the current pandemic, unfortunately, continues to flourish, clinicians worldwide are being confronted with new challenges. Post-COVID pulmonary fibrosis is one such dreadful sequela without any proven therapy. Many of the current antifibrotics have therapeutic potential for treating post-COVID fibrosis; long-term results are still awaited. We sincerely hope that issues associated with the current antifibrotics addressed in this commentary will open avenues for further research.

\section{OrCID}

Nitin Rai $\odot$ https://orcid.org/0000-0003-3351-4553

Dalim Kumar Baidya ๑ https://orcid.org/0000-0001-7811-7039

\section{References}

1. Carfi A, Bernabei R, Landi F, for the Gemelli Against COVID-19 PostAcute Care Study Group. Persistent symptoms in patients after acute COVID-19. JAMA 2020;324(6):603-605. DOI: 10.1001/jama.2020.12603.

(c) Jaypee Brothers Medical Publishers. 2021 Open Access This article is distributed under the terms of the Creative Commons Attribution 4.0 International License (https://creativecommons.org/licenses/by-nc/4.0/), which permits unrestricted use, distribution, and non-commercial reproduction in any medium, provided you give appropriate credit to the original author(s) and the source, provide a link to the Creative Commons license, and indicate if changes were made. The Creative Commons Public Domain Dedication waiver (http://creativecommons.org/publicdomain/zero/1.0/) applies to the data made available in this article, unless otherwise stated. 
2. Mo X, Jian W, Su Z, Chen M, Peng H, Peng P, et al. Abnormal pulmonary function in COVID-19 patients at time of hospital discharge. Eur Respir J 2020;55(6):2001217. DOI: 10.1183/13993003.01217-2020.

3. Wang J, Wang BJ, Yang JC, et al. Advances in the research of mechanism of pulmonary fibrosis induced by corona virus disease 2019 and the corresponding therapeutic measures. Zhonghua Shao Shang Za Zhi 2020;36(8):e006. DOI: 10.3760/cma.j.cn501120-20200307-00132.
4. Fernández Fabrellas E, Peris Sánchez R, Sabater Abad C, Juan Samper G. Prognosis and follow-up of idiopathic pulmonary fibrosis. Med Sci (Basel) 2018;6(2):51. DOI: 10.3390/medsci6020051

5. Kobayashi T, Tanaka K, Fujita T, et al. Bidirectional role of IL-6 signal in pathogenesis of lung fibrosis. Respir Res 2015;16:99. DOI: 10.1186/ s12931-015-0261-z. 